\title{
Thinning and Weighting of Planar/Conformal Arrays Considering Mutual Coupling Effects
}

\author{
You-Feng Cheng, Wei Shao, Ran Zhang, Xiao Ding, and Meng-Xia Yu \\ School of Physical Electronics, University of Electronic Science and Technology of China, Chengdu 610054, China \\ Correspondence should be addressed to You-Feng Cheng; juvencheng1377@gmail.com
}

Received 20 April 2016; Accepted 31 July 2016

Academic Editor: Christos Kalialakis

Copyright ( 2016 You-Feng Cheng et al. This is an open access article distributed under the Creative Commons Attribution License, which permits unrestricted use, distribution, and reproduction in any medium, provided the original work is properly cited.

Based on an improved active element pattern (AEP) technique, a novel effective method for sidelobe suppression considering mutual coupling $(\mathrm{MC})$ in planar and conformal sparse arrays is proposed in this paper. A thinning and weighting process that includes the thinning module, optimization module, and far-field calculation module is presented, and three key points, namely, the modified AEP modeling, far-field calculation of planar and conformal thinned arrays, and modified thinning strategy, are highlighted. As an effective optimization algorithm, the differential evolution algorithm (DEA) is adopted in order to achieve low sidelobe. Several numerical examples are shown to validate the consistency and effectiveness of the proposed synthesis approach. With the first use of the AEP technique for the synthesis of sparse arrays, the planar and conformal microstrip arrays with the desired array filling factor are studied to obtain the expected sidelobe level (SLL).

\section{Introduction}

The sparse or thinned array is considered as one of the main topics of innovative phased array antennas [1]. In terms of a thinned array, it is often acquired by removing some radiating elements from a uniform filled array. Compared with an equally spaced array, a thinned array has the nonuniform element distribution, high spatial resolution, and less elements, which leads to a reduction in the implementation and cost while maintaining the similar far-field characteristics [2]. However, since the interelement spacing may be larger than the Nyquist limit and the element locations are inhomogeneous, thinned arrays may have a deteriorative sidelobe level (SLL) which is a negative factor for the performance of arrays.

The sidelobe suppression is a very important issue in array design and even a great challenge for thinned arrays because the synthesis of a desired pattern of a thinned array is a nonlinear problem. Traditional excitations with tapered amplitude distributions, such as Dolph-Chebyshev and Taylor distributions, probably no longer gain excellent results [3]. Some researches on sidelobe reduction of thinned arrays have been carried out in the past few decades. In [3], an approach to suppress the sideband radiation pattern by rearranging the static excitation coefficients as well as the switch-on time intervals of each element was proposed. In [4-7], low-sidelobe synthesis of large planar array antennas was completed by using iterative Fourier transforms. Sum, difference, and shaped beams of nonuniformly spaced linear arrays were obtained through Poisson sum expansion of the array factor in [8]. In recent years, stochastic-optimization algorithms, such as the ant colony optimization (ACO) [9], particle swarm optimization (PSO) [10, 11], DEA [12], cat swarm algorithm [13-15], and genetic algorithm (GA) [16], have shown their excellent optimization capabilities and were extensively applied to the unequal-spacing array synthesis with the desired SLL.

In terms of the stochastic-optimization method, it is necessary to calculate the corresponding array pattern according to different array structures in the optimization process. Nevertheless, the abovementioned articles mainly focus on optimizing the excitation weights or element positions to reduce the sidelobe level and rarely consider the mutual coupling (MC) that has tremendous impacts on the array pattern when calculating the far-field characteristics. In practical applications, however, $\mathrm{MC}$ cannot be negligible yet when the interspacing of elements is considerable and the surrounding environment is complicated, especially for thinned arrays due to 


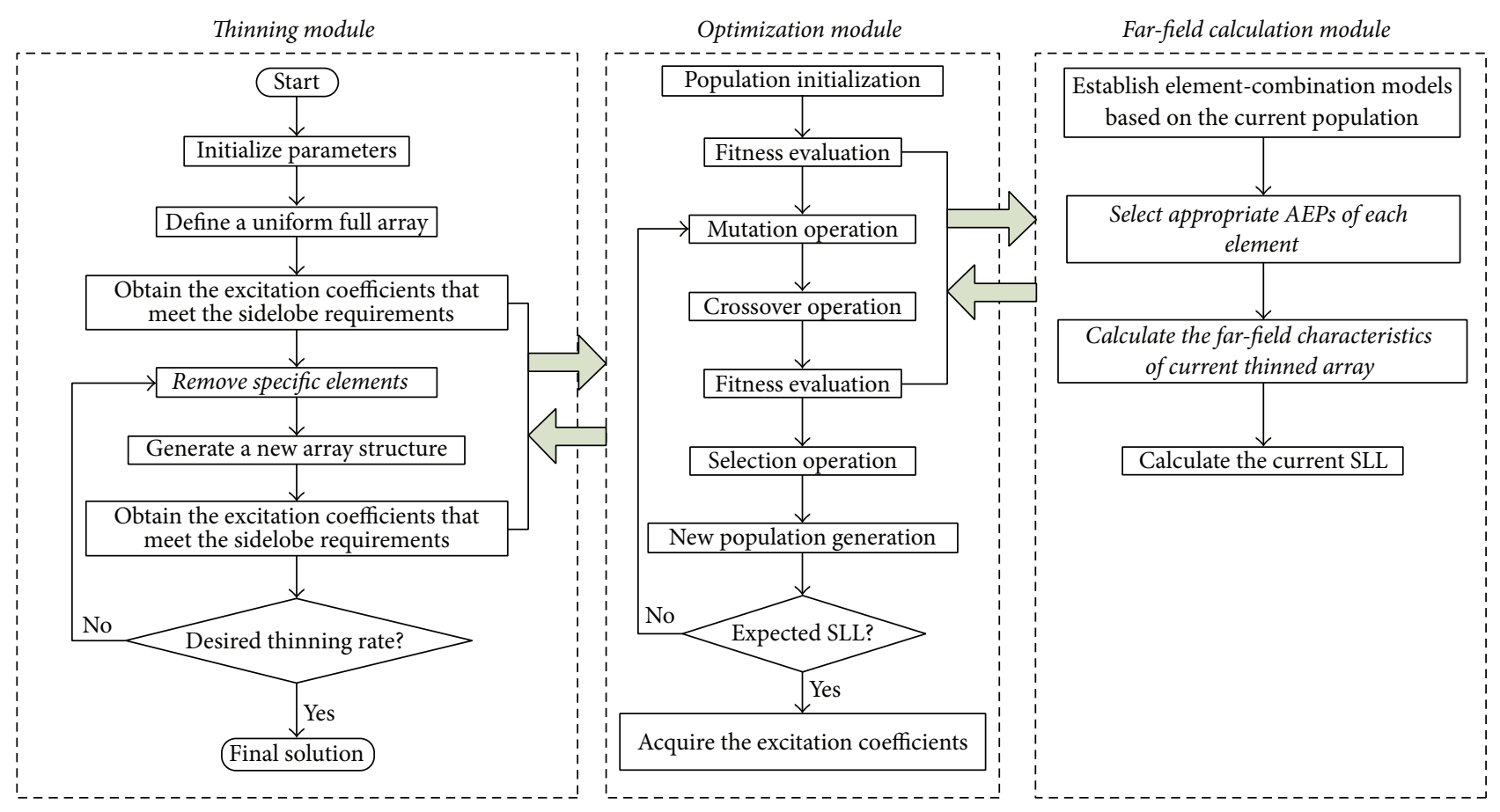

FIGURE 1: Flowchart of the thinning and weighting process.

their structural inhomogeneity [17]. In general, the full-wave electromagnetic simulation for a whole array can provide a credible solution. Reference [18] proposed a cosimulation optimization strategy through the MATLAB-API (Application Program Interface) files between the MATLAB software and a full-wave simulation software HFSS. For a thinned array design, however, the optimization process needs to call the simulation hundreds of times, and it is unrealistic to simulate the time-consuming whole array during the optimization process.

Various other methods are raised to eliminate or compensate the errors caused by the MC, such as the numerical analysis methods and active element pattern (AEP) modeling technique. The method of moments (MoM) is adopted to calculate the coupling matrix for evaluating the MC in [17, 19]. Although the MC is involved, this method is hard to be applied to large arrays due to the heavy computational burden. The AEP technique is simple, highly robust, and easy to implement [20,21]. References [22, 23] presented the AEP technique to calculate the array pattern that includes the MC effectively, especially for conformal arrays.

In this paper, a novel low-sidelobe synthesis method for planar and conformal arrays based on a modified AEP technique is proposed. Amplitude-only optimization is used in the synthesis process and several radiating elements are removed with a special array thinning strategy. Detailed AEP extraction steps of both planar and conformal thinned arrays are included in the far-field calculation. In our proposed method, the AEPs of all element-combination subarrays are calculated only once. Thus, the AEPs are just needed to be superposed without the full-wave simulation during the whole optimization process. Several numerical examples with expected array filling factors and SLLs prove that this technique can thin and weight the planar and conformal arrays with good performance.

This paper is organized as follows. In Section 2, the global synthesis process is summarized. The detailed description of AEP modeling of planar and conformal thinned arrays, array thinning strategy, and calculation of array patterns are presented in Section 3. In Section 4, several numerical examples are given to validate the accuracy of the far-field calculation of planar and conformal thinned arrays when considering MC effects and the consistency and effectiveness of the proposed synthesis approach. At last, Section 5 concludes all the work.

\section{Thinned Array Synthesis Process}

The flowchart of the global process for thinning and weighting of planar and conformal arrays is shown in Figure 1. There are three interrelated modules in the process, namely, the thinning module, optimization module, and far-field calculation module.

Initially, the excitation coefficients of a uniformly filled array, which meet the low-sidelobe demand, are obtained with the help of DEA in optimization module. To evaluate the fitness values, simultaneously, DEA needs to repeatedly call the far-field calculation module in which the AEP technique is used to calculate the far-field characteristics of the array based on the optimized results.

Then, a modified thinning scheme is applied here to remove some elements from the uniform array according to a given threshold of the excitation coefficients. The newly generated thinned array also needs to be optimized in the optimization module to get its appropriate excitation 


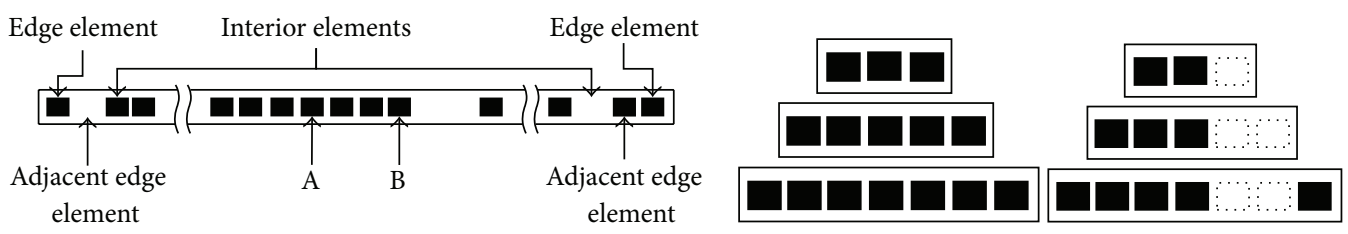

(a)

(b)
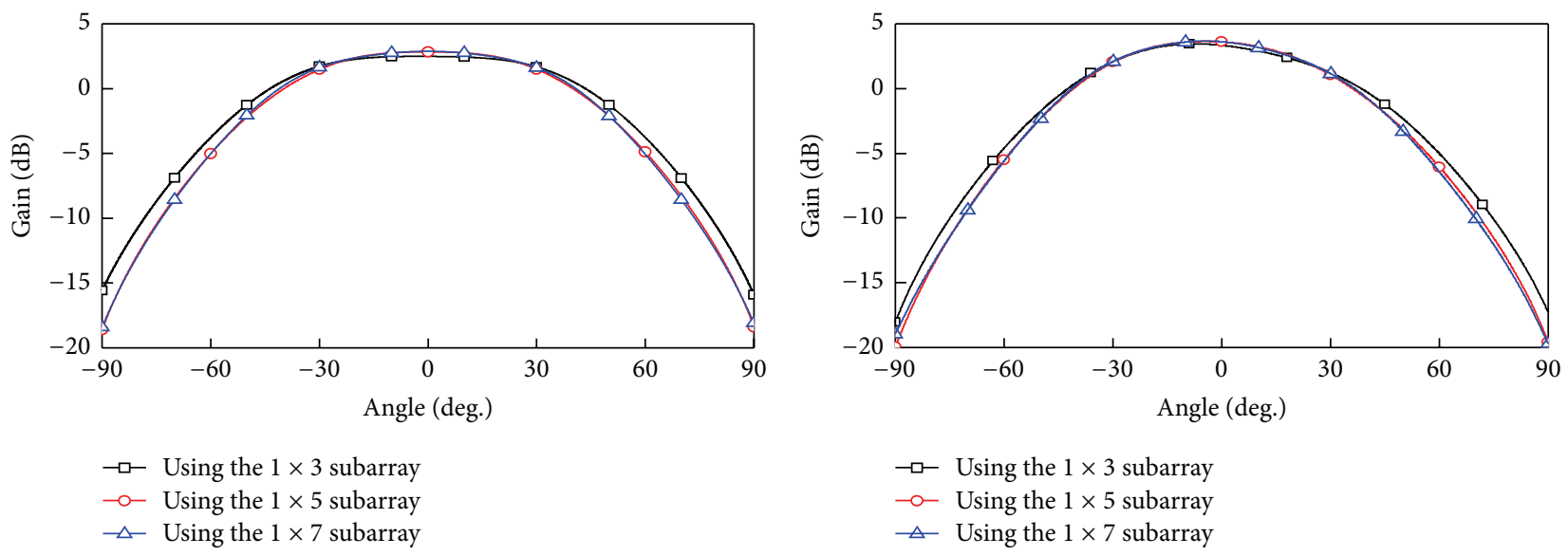

(c)

FIgure 2: Patch subarrays and their corresponding AEPs. (a) Top view of a thinned linear patch array, (b) subarrays used to extract the AEPs of elements A and B, where a dotted rectangle denotes a position without element and a black rectangle denotes a position with element, and (c) AEPs of elements A and B.

coefficients to meet the low-sidelobe demand as mentioned above.

Besides the sidelobe, the array filling factor, which is defined as the ratio between the element number of the current thinned array and that of the uniformly filled array, is another important objective for the thinning design. If the current thinned array with the desired sidelobe level satisfies the given requirement of the filling factor, then the whole synthesis process is finished.

The AEP modeling of arbitrary planar and conformal thinned arrays, the calculation of array patterns, and the modified thinning strategy, which correspond to the italic fonts in Figure 1, are emphasized in detail in Section 3.

In the traditional synthesis process, the gain patterns of different thinned array structures need to be calculated hundreds of times. The calculation is very time-consuming if a full-wave algorithm is used to simulate an array with all elements. In the proposed method, however, the AEPs of subarrays need to be extracted only once in the beginning. To calculate the far-field characteristics, the superposition of the AEPs of the subarrays is involved instead of the full-wave simulation for the whole array.

\section{Modified AEP Extraction Method, Far-Field Analysis of Thinned Arrays, and Modified Thinning Strategy}

3.1. Modified AEP Extraction of Thinned Arrays. According to $[22,23]$, the elements of an arbitrary array can be divided into three parts in the AEP model, namely, the edge elements, the adjacent edge elements, and the interior elements. Therefore, its far field can be written as

$$
\mathbf{E}_{\text {total }}=\mathbf{E}_{e}+\mathbf{E}_{a e}+\mathbf{E}_{i}
$$

where $\mathbf{E}_{e}, \mathbf{E}_{a e}$, and $\mathbf{E}_{i}$ are the vector superpositions of AEPs of all the edge, adjacent edge, and interior elements, respectively.

In order to describe the AEP extraction for thinned arrays in detail, as shown in Figure 2(a), a linear array which is thinned from a patch array with the regular lattice of $N$ positions spaced by a uniform distance is used as an example. For each element, the MC mainly depends on its neighboring elements and the effects of other elements can be negligible. In other words, only a few neighboring elements are involved in calculating its far-field pattern.

To obtain the accurate AEPs of elements A and B, subarrays with the size of $1 \times 3,1 \times 5$, and $1 \times 7$, as shown in Figure 2(b), are calculated, respectively. Here, the interelement spacing is about $0.5 \lambda_{0}$, where $\lambda_{0}$ is the freespace wavelength. It can be found from Figure 2(c) that the AEP of the $1 \times 5$ subarray is in good agreement with that of the $1 \times 7$ subarray. In other words, the AEP of the $1 \times 5$ subarray possesses sufficient accuracy to calculate $\mathbf{E}_{i}$. Similarly, for the edge elements and the adjacent edge elements, the AEPs of the $1 \times 3$ subarray and the $1 \times 4$ subarray are applied to calculate $\mathbf{E}_{e}$ and $\mathbf{E}_{a e}$, respectively.

However, unlike the uniformly distributed array, element states (positions with or without an element) of a thinned array are unknown and there are a variety of possibilities. For a certain element in the thinned array, the distributions of its 
neighboring elements are various. Therefore, extracting the AEPs of all element combinations is indispensable.

Method of exhaustion in the subarray is used here. According to the abovementioned five-element subarray, for each edge element in Figure 2(a), the element combinations with the two neighboring positions are of $2^{2}$ kinds, and for each interior element, the element combinations with the four neighboring positions are of $2^{4}$ kinds. From the AEP calculations, it can be found that the element-combination models of adjacent edge elements are contained in corresponding interior element models when studying the practical array environment. Thus, there are $2 \times 2^{2}+2^{4}=24$ kinds of permutations and combinations of possibilities. The AEPs of subarrays can be obtained by simulation of commercial software or measurement of fabricated prototypes.

Generally, the mutual coupling intensity between elements varies with different interelement spacing $d$ in an array. Thus, the corresponding size of extracted subarrays may be different. Based on the abovementioned analysis process, the subarray size and element-combination number can be determined by different $d$. For the typical values of $d$ in a linear patch array, the types of subarrays and AEP numbers required to be extracted are listed in Table 1. The shorter the distance between elements is, the stronger the mutual coupling is. Therefore, when $d$ is short, more elements are involved to extract the AEPs to satisfy the accuracy requirement.

Note that the AEP extraction of a conformal thinned array is almost the same as that of a planar thinned array. The elements of an arbitrary array can be also divided into the edge elements, adjacent edge elements, and interior elements [23]. Then, all the possibilities of the subarray structures are considered and their AEPs are extracted for the far-field analysis. Different from the cases of planar thinned arrays, the required minimum subarray size of a conformal thinned array depends not only on the interelement spacing but also on the curvature of the supporting platform. This method suits all the thinned array conformed on a regular platform, such as cylindrical, parabolic cylindrical, elliptic cylinder, and spherical platforms. In terms of thinned arrays conformed on an irregular platform, the subarray cannot represent the whole array, and thus it is hard to apply this method to calculate the far-field characteristics. It is worth noting that, for the given numerical examples, this method is efficiently applicable.

3.2. Analysis of Planar and Conformal Thinned Arrays. By the principle of superposition of AEPs, the far-field pattern radiated by a thinned linear array can be expressed as

$$
E_{\text {total }}=\sum_{n=1}^{N} I_{n} \sqrt{G_{a}^{n}(\theta, \varphi)} e^{\left(j k d_{n} \cos \varphi \sin \theta+j \psi\right)},
$$

where $I_{n}$ is the amplitude of the $n$th excitation current, $d_{n}$ is the distance between the $n$th element and the coordinate origin, $\psi$ is the phase term of the $n$th excitation current, and $G_{a}^{n}(\theta, \varphi)$ is the active element gain pattern of the $n$th element which can be extracted from the full-wave simulation or measurement. It is worth noting that the AEPs used here are extracted with the method presented in Section 3.
TABLE 1: Types of subarrays and their AEP numbers.

\begin{tabular}{lcccc}
\hline \multirow{2}{*}{} & \multicolumn{3}{c}{ Type of subarrays } & AEP \\
& Edge & Adjacent edge & Interior & number \\
\hline $0.33 \lambda_{0} \sim 0.45 \lambda_{0}$ & $1 \times 4$ & $1 \times 6$ & $1 \times 7$ & 80 \\
$0.45 \lambda_{0} \sim 0.64 \lambda_{0}$ & $1 \times 3$ & $1 \times 4$ & $1 \times 5$ & 24 \\
$0.64 \lambda_{0} \sim 0.8 \lambda_{0}$ & $1 \times 2$ & $1 \times 3$ & $1 \times 3$ & 8 \\
$>0.8 \lambda_{0}$ & \multicolumn{3}{c}{ Mutual coupling effects can be ignored. } \\
\hline
\end{tabular}

Thus, the total gain pattern of a thinned linear array can be given by

$$
F_{G}=20 \log \left(\frac{E_{\text {total }}}{\sqrt{\sum_{n=1}^{N} I_{n}^{2}}}\right) .
$$

Unlike planar thinned arrays, the AEPs of conformal thinned arrays point to different directions and cannot be simply substituted into (2) to calculate the total field since they are extracted in a local coordinate system. Therefore, it is necessary to change the AEPs from the local coordinate system into a global one, and a local rotating coordinate system is introduced to establish linkages between the two coordinate systems [23]. The major processing steps can be summarized as follows.

Step 1. Establish the local coordinate system and local rotating coordinate system of the $n$th element, and extract its AEP $G_{n}\left(\theta_{n}^{l}, \varphi_{n}^{l}\right)$ from HFSS simulation, where the superscript " $l$ " represents the local coordinate system of the element. For each element in a conformal thinned array, the extraction of its AEP is the same as a linear thinned array described in Section 2.

Step 2. Transform $G_{n}\left(\theta_{n}^{l}, \varphi_{n}^{l}\right)$ into $G_{n}\left(\theta_{n}^{r}, \varphi_{n}^{r}\right)$, where the superscript " $r$ " represents the local rotating coordinate system of the element $[22,23]$. The element-combination models are established here with the same method as that introduced in Section 2.

Step 3. Express the total far-field pattern as a superposition of all AEPs:

$$
\begin{gathered}
\mathbf{E}_{\text {total }}(\theta, \varphi)=\widehat{\theta}\left\{\sum _ { n = 1 } ^ { N } \mathbf { I } _ { n } \left[\sqrt{G_{n \theta_{n}^{r}}\left(\theta_{n}^{r}, \varphi_{n}^{r}\right)}\right.\right. \\
\cdot \frac{\sin \theta \cos \theta_{n}-\cos \theta \sin \theta_{n} \cos \left(\varphi-\varphi_{n}\right)}{\sin \theta_{n}^{r}} \\
\left.-\sqrt{G_{n \theta_{n}^{r}}\left(\theta_{n}^{r}, \varphi_{n}^{r}\right)} \frac{\sin \theta_{n} \sin \left(\varphi-\varphi_{n}\right)}{\sin \theta_{n}^{r}}\right] \\
\left.\cdot e^{j k R_{n}\left[\sin \theta \sin \theta_{n} \cos \left(\varphi-\varphi_{n}\right)+\cos \theta \cos \theta_{n}\right]}\right\}+\widehat{\varphi}\left\{\sum_{n=1}^{N} \mathbf{I}_{n}\right. \\
\cdot\left[\sqrt{G_{n \theta_{n}^{r}}\left(\theta_{n}^{r}, \varphi_{n}^{r}\right)}\right.
\end{gathered}
$$




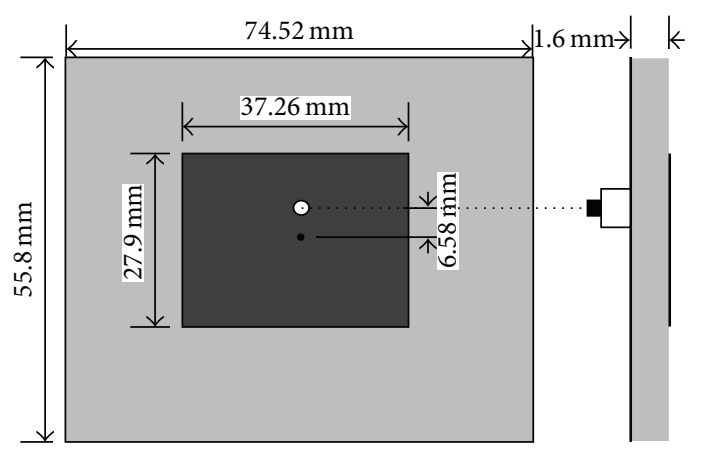

(a)

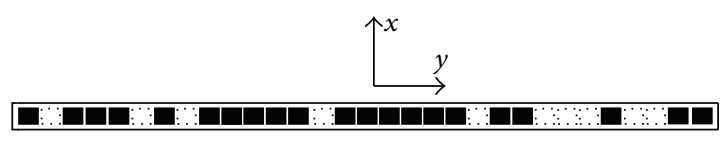

(b)

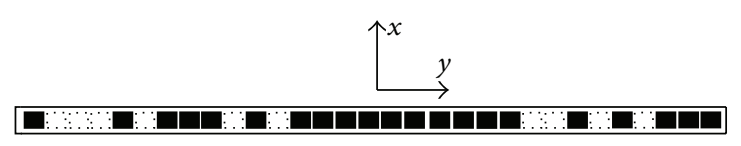

(c)

FIgURE 3: Geometries of linear thinned arrays. (a) Structure of the patch antenna element. (b) Top view of array 1. (c) Top view of array 2.

$$
\begin{aligned}
& \cdot \frac{\sin \theta \cos \theta_{n}-\cos \theta \sin \theta_{n} \cos \left(\varphi-\varphi_{n}\right)}{\sin \theta_{n}^{r}} \\
& \left.+\sqrt{G_{n \theta_{n}^{r}}\left(\theta_{n}^{r}, \varphi_{n}^{r}\right)} \frac{\sin \theta_{n} \sin \left(\varphi-\varphi_{n}\right)}{\sin \theta_{n}^{r}}\right] \\
& \left.\cdot e^{j k R_{n}\left[\sin \theta \sin \theta_{n} \cos \left(\varphi-\varphi_{n}\right)+\cos \theta \cos \theta_{n}\right]}\right\},
\end{aligned}
$$

where $\mathbf{I}_{n}$ is the excitation current of the $n$th element, $G_{n \theta_{n}^{r}}\left(\theta_{n}^{r}, \varphi_{n}^{r}\right)$ is the gain pattern of the $n$th element of the local rotating coordinate system obtained in Step 2, and $\theta_{n}$ and $\varphi_{n}$ determine the $n$th element position with respect to the global coordinate origin. Once all the position parameters and gain patterns of a practical nonlinear thinned array are known, the array pattern can be calculated with (3) and (4).

3.3. Modified Thinning Strategy of the Synthesis Procedure. Normally, an element with a small amplitude weight makes a minor contribution to the array pattern and it can be ignored. In this way, in the thinning module of Figure 1, the radiating elements with amplitude weights which are lower than a predefined threshold will be removed, and then a new array distribution is generated. At the same time, since the array aperture determines the spatial resolution, the first and the last elements must be retained regardless of the values of their excitation coefficients.

Unlike the complex termination conditions in [16], the iteration of thinning module stops when the filling factor is smaller than a desired value in our method. Then, the thinning module needs to call the optimization module for the last time, and therefore the excitation coefficients are possibly lower than the threshold.

DEA is utilized in the optimization process which changes the population distribution and finds the best fitness value through mutation, crossover, and selection operations.
Self-adapting control parameters, that is, the mutation parameter and crossover parameter, prevent the algorithm from being easily trapped into local optima and ensure the convergence rate [24].

\section{Numerical Examples}

4.1. Far-Field Analysis of Thinned Arrays. In order to examine the feasibility, validity, and accuracy of the proposed analysis method, the results of two examples are presented. Figures 3(b) and 3(c) show two different arrays thinned from the same uniform filled array with 31 elements lying along the $y$-axis. A dotted rectangle denotes a position without the element and a black rectangle denotes a position with the element, respectively. The uniform filled array is mounted on the dielectric substrate with a thickness of $h=1.6 \mathrm{~mm}$ and a relative permittivity of 4.4. As shown in Figure 3(a), each element is a rectangle patch antenna excited with the same amplitude and its position is uniformly spaced from its neighbors by a distance of $d=0.46 \lambda_{0}$, where $\lambda_{0}$ is the free-space wavelength corresponding to an operating frequency of $2.45 \mathrm{GHz}$.

Figures 4(a) and 4(b) illustrate the radiation patterns of the two thinned arrays in Figures 3(b) and 3(c) from the classical method of pattern multiplication, full-wave simulation with HFSS software, and our proposed method. At the top of Figures 4(a) and 4(b), " 1 " indicates that there is an element on the corresponding position and " 0 " indicates that there is no element on the position. The performance details are listed in Table 2. Apparently, the pattern parameters such as the peak sidelobe level (PSL), gain, and half-power beamwidth (HPBW) from our method are in good agreement with those from HFSS simulation, and the results of the classical method are not accurate enough since the MC effects are not involved.

It is worth noting that these AEPs are obtained through the HFSS simulation of some subarrays. The total time spent on the AEP extraction and the vector superposition of the AEPs is far shorter than the full-wave simulation of the whole 
TABLE 2: Gain pattern performance of planar thinned arrays.

\begin{tabular}{|c|c|c|c|c|c|c|c|c|}
\hline & \multicolumn{4}{|c|}{ Array 1} & \multicolumn{4}{|c|}{ Array 2} \\
\hline & Gain (dBi) & HPBW (deg) & PSL (dB) & Runtime & Gain $(\mathrm{dBi})$ & HPBW (deg) & PSL (dB) & Runtime (s) \\
\hline Classical & 17.19 & 4.10 & -14.43 & & 17.19 & 4.07 & -14.11 & \\
\hline AEP & 16.62 & 3.72 & -15.08 & $33.6 \mathrm{~min}$ & 16.64 & 3.67 & -15.46 & $33.6 \mathrm{~min}$ \\
\hline HFSS & 16.58 & 3.69 & -15.74 & 12.4 hours & 16.80 & 3.69 & -15.47 & 11.9 hours \\
\hline
\end{tabular}

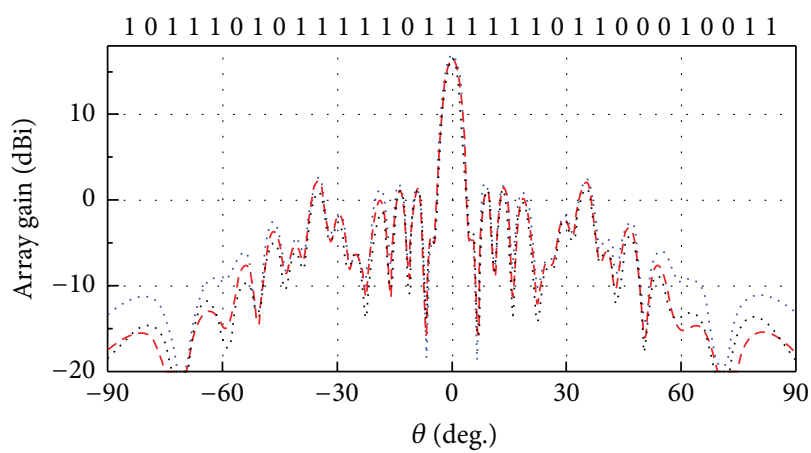

-.... Classical method
-- AEP technique
$\ldots .$. HFSS

(a)

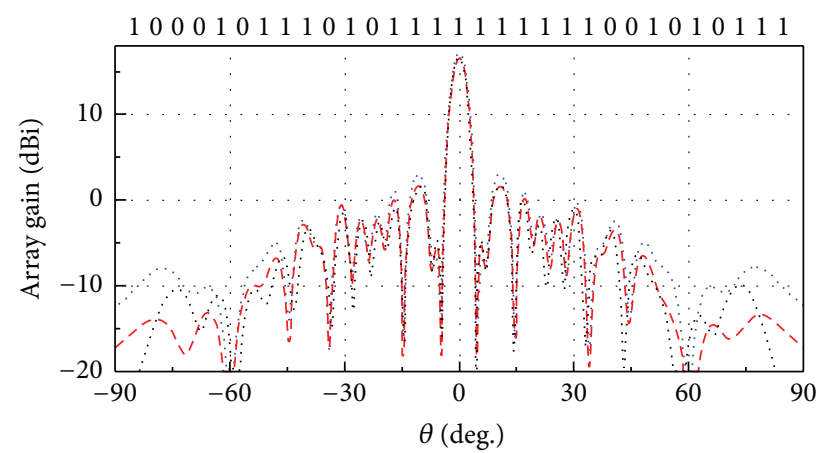

… Classical method
--- AEP technique
… HFSS

(b)

FIGURE 4: Gain patterns of the planar thinned arrays. (a) Thinned array 1. (b) Thinned array 2.

thinned array. The runtimes of the proposed method are mainly spent on the simulations of the subarrays. From Table 2, it can be found that the runtimes of the proposed method (both 33.6 minutes) are far shorter than the HFSS simulations (12.4 and 11.9 hours). Furthermore, the runtime of the proposed method is independent of the array size, while that of the HFSS simulation dramatically rises with the increase of the array size. Simulations are implemented on a PC equipped with an Intel Core i5-3470 $(3.2 \mathrm{GHz})$ processor and 64 GB RAM.

Another two conformal thinned arrays which are thinned from the same full array with 15 elements are shown in Figure 5. The filled array lies on a curve of radius of $R=$ $10 \lambda_{0}$, where $\lambda_{0}$ is the free-space wavelength at the operating frequency of $2.35 \mathrm{GHz}$. The patch antenna element mounted on an FR4 substrate with a thickness of $1.6 \mathrm{~mm}$ and a relative permittivity of 4.4 is shown in Figure 5(a). The interelement spacing is $d=0.6 \lambda_{0}$. In Figures 5(b) and 5(c), the black strips on the curve depict the positions in which elements exist. It should be mentioned that, for each port excitation, it is necessary to compensate a phase, and the compensation of the $n$th element is

$$
\varphi_{n}=k \mathbf{R}_{n} \cdot \widehat{\mathbf{r}}_{0} \pm 2 n \pi,
$$

where $k$ is the wave number, $\mathbf{R}_{n}$ is the position vector from the origin to the center of the $n$th element, and $\widehat{\mathbf{r}}_{0}$ is the unit vector of the expected beam pointing direction.

The gain patterns of these two thinned arrays can be calculated and are shown in Figures 6(a) and 6(b). From the performance details listed in Table 3, it can be found that the PSL, gain, and HPBW from our method are all in good agreement with those from full-wave simulation. Moreover, the runtimes of the proposed method (146 minutes) are shorter than those of the HFSS simulations (15.8 and 15.7 hours).

\subsection{Thinning and Weighting of Planar and Conformal Arrays.}

Two groups of numerical examples are shown here to certify the feasibility of the proposed method for sidelobe reduction of planar and conformal thinned arrays with a given filling factor, respectively.

For the first group of examples, three designs of lowsidelobe sparse linear arrays with the given filling factors are shown here. The sparse arrays are thinned from a filled array with 35 elements located at the $y$-axis, and their structural characteristics and interelement spacing are the same as those in Figure 3. The SLL is ordered to meet the requirements by optimizing the excitation coefficients and locations where the elements are removed. For the first array which is denoted as LA-I, ten elements need to be removed and the PSL is required lower than $-20 \mathrm{~dB}$. Likewise, the second array is denoted as LA-II, and it is ordered to remove ten elements and to have a PSL lower than $-20 \mathrm{~dB}$. Note that the LA-II scans its main beam to $+20^{\circ}$, and a phase difference of $-57.1926^{\circ}$ is used. The third array LA-III is designed to have a filling factor of $57 \%$ and a PSL of $-20 \mathrm{~dB}$.

In DEA, the initial scaling factor $(F)$ and the initial crossover rate (CR) are chosen to be 0.5 and 0.9 , respectively. The population parameters are selected as Pop_Size (population size $)=100$, Dim (population dimension) $=31$, Pop_Min (minimum individual $)=0$, and Pop $\_$Max $($maximum individual $)=$ 1. Therefore, the excitation coefficients vary between 0 and 1. The maximum numbers of iterations in thinning module 
TABLE 3: Gain pattern performance of conformal thinned arrays.

\begin{tabular}{lcccccccc}
\hline & \multicolumn{4}{c}{ Array 1} & & \multicolumn{3}{c}{ Array 2} \\
& Gain $(\mathrm{dBi})$ & HPBW $(\mathrm{deg})$ & PSL $(\mathrm{dB})$ & Runtime & Gain $(\mathrm{dBi})$ & HPBW $(\mathrm{deg})$ & PSL (dB) & Runtime \\
\hline AEP & 12.36 & 5.75 & -9.32 & 246 min & 16.64 & 6.29 & -10.07 & 246 min \\
HFSS & 12.18 & 5.73 & -9.54 & 16.8 hours & 16.80 & 6.22 & -10.72 & 15.7 hours \\
\hline
\end{tabular}

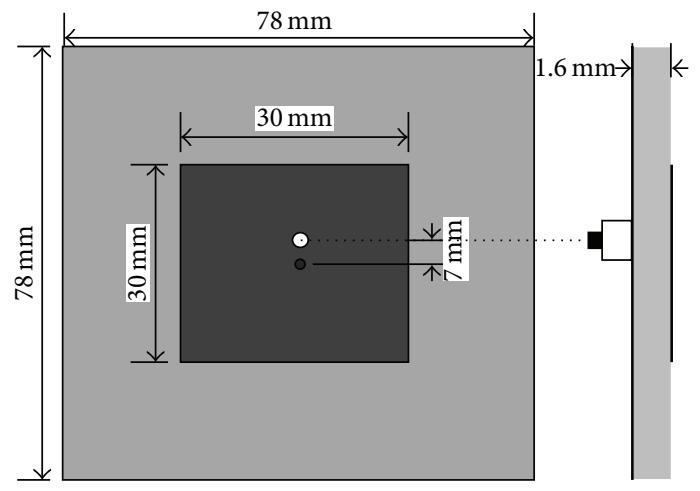

(a)

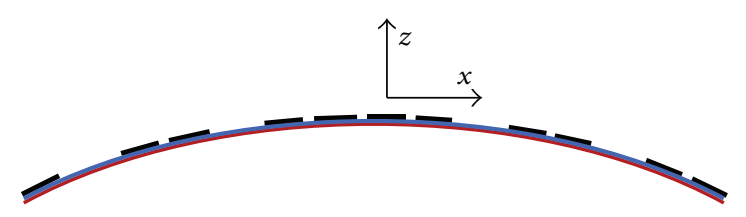

(b)

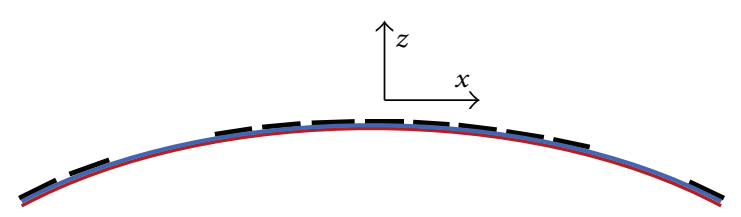

(c)

FIGURE 5: Geometries of nonlinear thinned arrays. (a) Structure of the patch antenna element, (b) side view of array 1, and (c) side view of array 2.

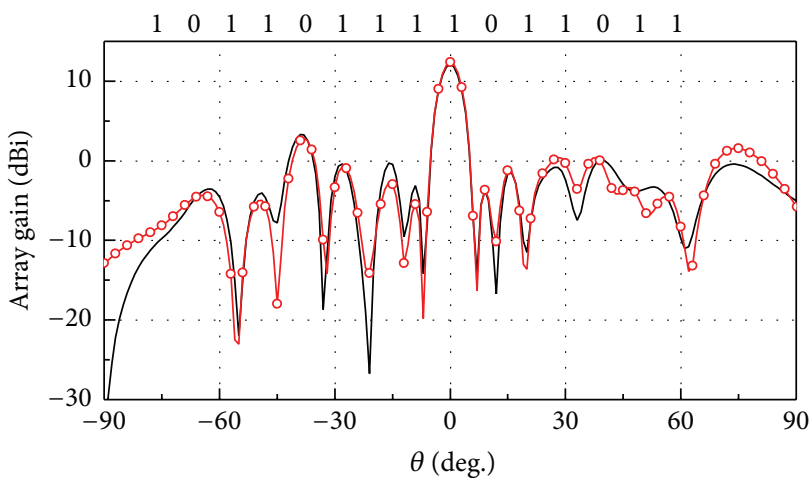

— AEP technique $\multimap$ HFSS

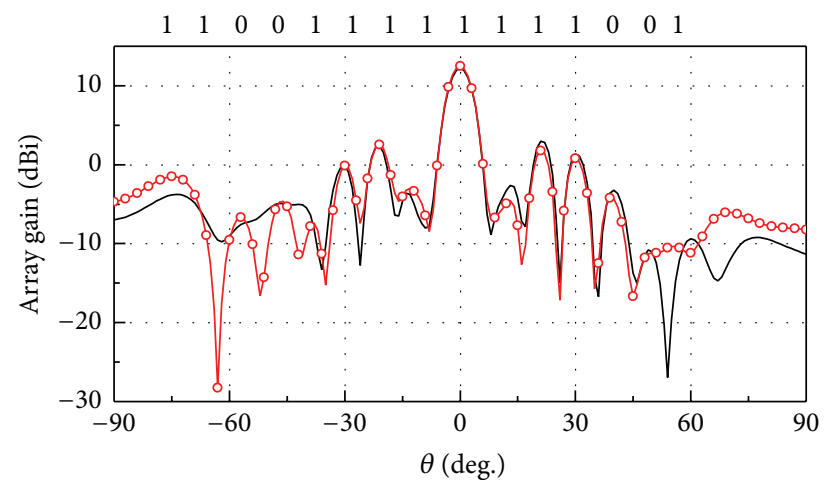

- AEP technique $\rightarrow$ HFSS

(a)

(b)

Figure 6: Gain patterns of conformal thinned arrays. (a) Thinned array 1. (b) Thinned array 2.

and optimization module are 100 and 300, respectively. It is worth noting that the selection of ar is very important and here it is chosen as 0.25 . Generally, the larger ar is, the less the computational time is spent with the worse performance of the array. However, a small value of ar will lead to more computational time. Therefore, there is a tradeoff about the selection of $a r$.

After the optimization, the excitation coefficients and the gain patterns of the thinned and weighted arrays are shown in
Table 4 and Figure 7, respectively. The excitation coefficients of elements being zero indicate that the elements have been removed. The first and last elements are retained in order to keep the array aperture size.

It can be observed from Figure 7 that the optimized gain patterns from the proposed AEP method show good agreement with those from the HFSS simulation, and the optimized results all meet the design requirements. From the element distributions of the three arrays, it can be found that 


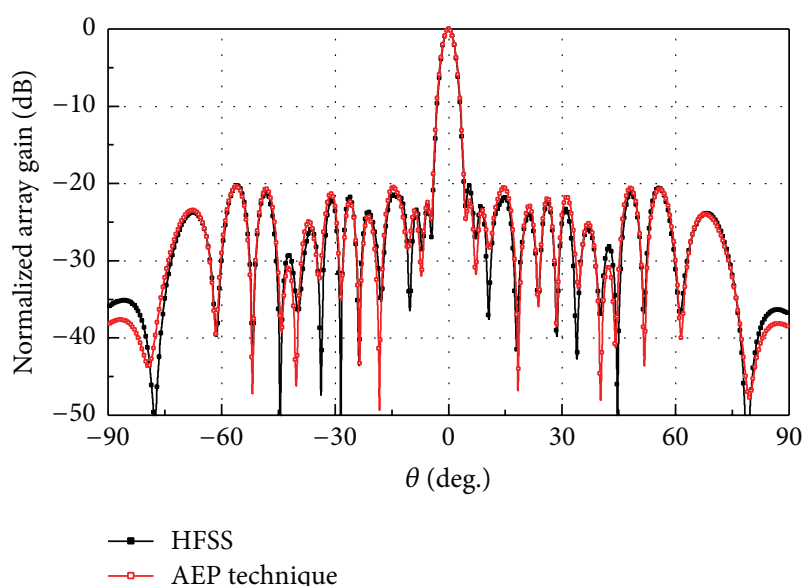

(a)

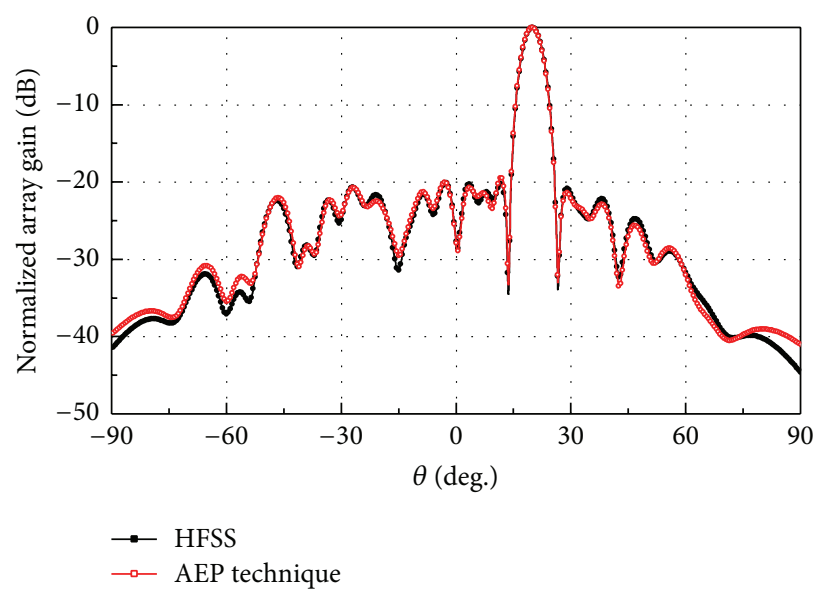

(b)

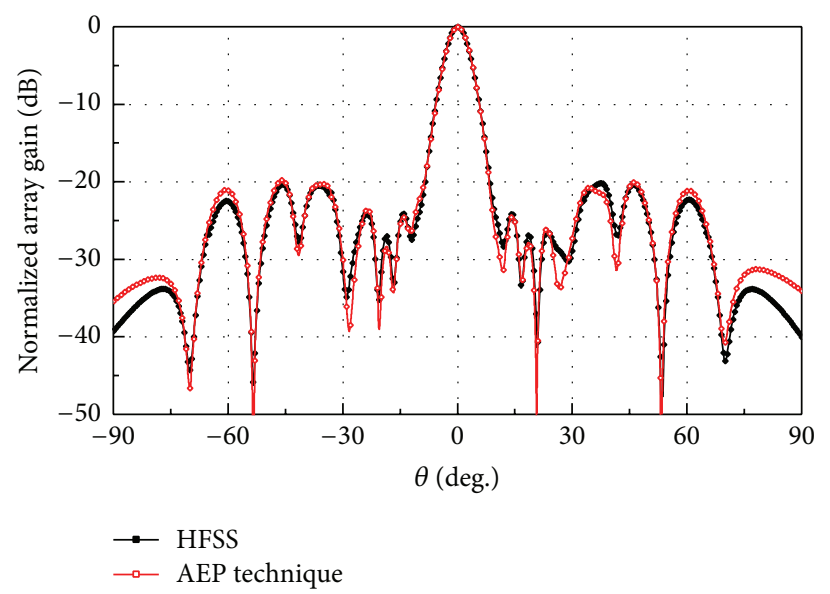

(c)

FIGURE 7: Optimized gain patterns of the three linear sparse arrays. (a) LA-I. (b) LA-II. (c) LA-III.

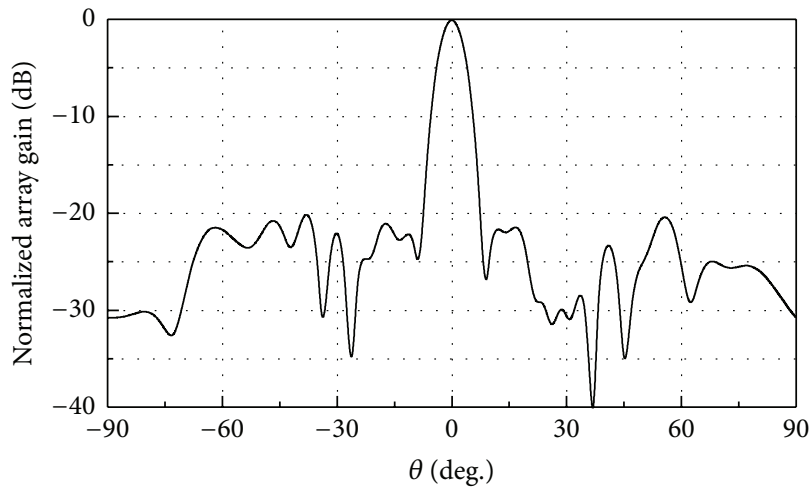

(a)

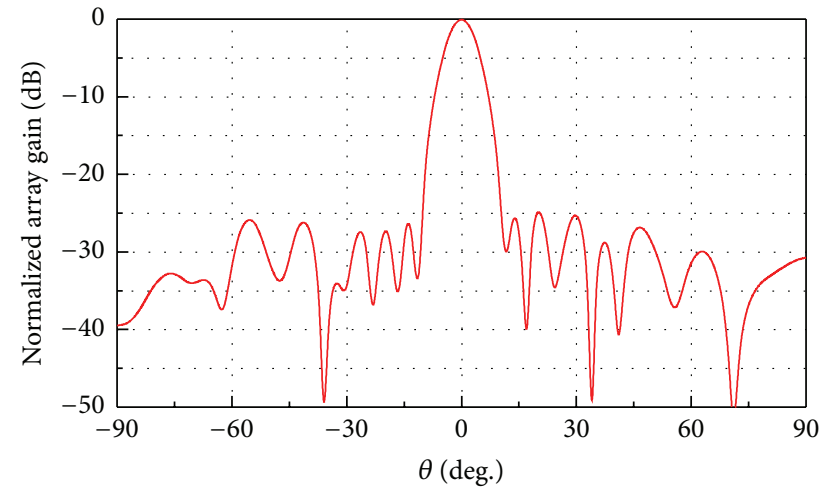

(b)

FIGURE 8: Optimized gain patterns of the two conformal sparse arrays. (a) CA-I. (b) CA-II.

the array elements are mainly distributed in the middle region due to the amplitude tapering for low sidelobes. In addition, with the decrease of the array filling factor, more elements located in the both ends are removed, and the HPBW has a certain broadening. When the main-beam direction tilts from $0^{\circ}$ to $+20^{\circ}$, more elements on the left side are removed.
For the second group of examples, another two designs of two conformal sparse arrays (CA-I and CA-II) with sidelobe reduction are shown here. The sparse arrays are thinned from a filled array with 22 elements in the xoz plane, and their structural characteristics and interelement spacing are the same as those in Figure 5. Both sparse arrays are required to 
TABLE 4: Element distributions and optimized amplitudes of the three linear sparse arrays.

\begin{tabular}{|c|c|c|}
\hline \multirow[b]{2}{*}{ LA-I } & Element distribution & 10100101111111111111110101101011001 \\
\hline & Amplitude (A) & $\begin{array}{l}0.6557,0.0000,0.9034,0.0000,0.0000,0.9013,0.0000,0.6198,0.3560,0.8782, \\
0.7728,0.7587,0.6465,0.7774,0.7279,0.4993,0.6422,0.7209,0.7060,0.6418,0.6128 \text {, } \\
0.5683,0.0000,0.7931,0.0000,0.7181,0.5719,0.0000,0.6057,0.0000,0.4705,0.3474 \text {, } \\
0.0000,0.0000,0.2860\end{array}$ \\
\hline \multirow[b]{2}{*}{ LA-II } & Element distribution & 10000001111111111111111111110101001 \\
\hline & Amplitude (A) & $\begin{array}{l}0.0309,0.0000,0.0000,0.0000,0.0000,0.0000,0.0000,0.4502,0.4853,0.5708, \\
0.5629,0.5615,0.5163,0.5429,0.4734,0.6313,0.7057,0.8532,0.6045,0.8745,0.9327, \\
0.7237,0.3547,0.8635,0.8186,0.6485,0.6532,0.2986,0.0000,0.6638,0.0000,0.3997 \text {, } \\
0.0000,0.0000,0.0704\end{array}$ \\
\hline \multirow[b]{2}{*}{ LA-III } & Element distribution & 10000001001010111111111011010000001 \\
\hline & Amplitude (A) & $\begin{array}{l}0.1427,0.0000,0.0000,0.0000,0.0000,0.0000,0.0000,0.4617,0.0000,0.0000, \\
0.6078,0.0000,0.6175,0.0000,0.7166,0.6608,0.7299,0.6138,0.9983,0.7057,0.6652, \\
0.5778,0.9595,0.0000,0.5271,0.3010,0.0000,0.3281,0.0000,0.0000,0.0000, \\
0.0000,0.0000,0.0000,0.0866\end{array}$ \\
\hline
\end{tabular}

TABLE 5: Element distributions and optimized amplitudes of the two conformal sparse arrays.

\begin{tabular}{lcl}
\hline & Element distribution & 10010111111111111110001 \\
CA-I & Amplitude (A) & $0.1109,0.0000,0.0000,0.5941,0.0000,0.6512,0.5550,0.6988,0.8119,0.9018,0.9985$, \\
& $0.8641,0.8679,0.8093,0.8622,0.6207,0.7001,0.2151,0.0000,0.0000,0.0000,0.2075$ \\
\hline \multirow{2}{*}{ CA-II } & Element distribution & 10001111111111111101001 \\
& & $0.1327,0.0000,0.0000,0.0000,0.1096,0.4232,0.3588,0.6688,0.7460,0.9572,0.9495$, \\
& Amplitude (A) & $0.9739,0.9191,0.6871,0.5961,0.4178,0.3381,0.0000,0.1015,0.0000,0.0000,0.0625$ \\
\hline
\end{tabular}

remove six elements. For CA-I, the PSL should be lower than $-20 \mathrm{~dB}$. For CA-II, the PSL should be lower than $-25 \mathrm{~dB}$.

The DEA parameters are all the same with the several previous examples and ar is also selected as 0.25 . Corresponding parameters of DEA are listed as Pop_Size $=100$, $\operatorname{Dim}=31$, Pop_Min $=0$, Pop_Max $=1, \mathrm{CR}=0.9$, and $F=0.5$. The maximum numbers of iterations in thinning module and optimization module are 100 and 300, respectively.

Figure 8 shows the obtained gain patterns of the two thinned and weighted conformal arrays, and Table 5 presents the element distributions and input amplitudes. It can be found that both the arrays meet the requirements of the PSL and filling factor. The removed elements are mainly concentrated in the two ends of the array due to the amplitude tapering.

\section{Conclusion}

This paper describes a thinning and weighting process that includes the thinning module, optimization module, and farfield calculation module for sidelobe suppression. An efficient AEP extraction technique, which considers the MC accurately, is introduced for thinned arrays. Based on the technique, the far-field calculations of both planar and conformal thinned arrays are presented. Several numerical examples that compare the results of the proposed method with fullwave simulation verify the validity and accuracy of the AEP technique. With the first use of the AEP technique for the synthesis of thinned arrays, the synthesis process is time-saving to realize the sidelobe reduction. Then, several examples of the low-sidelobe synthesis of thinned arrays are introduced to show that the synthesis process is able to synthesize planar and conformal thinned arrays with the given low sidelobes and filling factors.

\section{Competing Interests}

The authors declare that they have no competing interests.

\section{Acknowledgments}

This work was supported by the National Natural Science Foundation of China (nos. 61401065 and 61471105) and 973 Project (no. 613273).

\section{References}

[1] G. Toso and R. Mailloux, "Guest editorial for the special issue on innovative phased array antennas based on non-regular lattices and overlapped subarrays," IEEE Transactions on Antennas and Propagation, vol. 62, no. 4, pp. 1546-1548, 2014.

[2] F. Tokan and F. Güne, "Pareto optimal synthesis of the linear array geometry for minimum sidelobe level and null control during beam scanning," International Journal of $R F$ and Microwave Computer-Aided Engineering, vol. 20, no. 5, pp. 557566, 2010.

[3] L. Zhang, Y.-C. Jiao, B. Chen, and H. Li, "Orthogonal genetic algorithm for planar thinned array designs," International Journal of Antennas and Propagation, vol. 2012, Article ID 319037, 7 pages, 2012. 
[4] W. P. M. N. Keizer, "Fast low-sidelobe synthesis for large planar array antennas utilizing successive fast fourier transforms of the array factor," IEEE Transactions on Antennas and Propagation, vol. 55, no. 3, pp. 715-722, 2007.

[5] W. P. M. N. Keizer, "Large planar array thinning using iterative FFT techniques," IEEE Transactions on Antennas and Propagation, vol. 57, no. 10, pp. 3359-3362, 2009.

[6] X. Wang, Y. Zhou, and Y. Wang, "An improved antenna array pattern synthesis method using fast fourier transforms," International Journal of Antennas and Propagation, vol. 2015, Article ID 316962, 9 pages, 2015.

[7] W. Li, Y. Hei, J. Yang, and X. Shi, "Fractal-based thinned planararray design utilizing iterative FFT technique," International Journal of Antennas and Propagation, vol. 2012, Article ID 329648, 7 pages, 2012.

[8] H. Oraizi and M. Fallahpour, "Sum, difference and shaped beam pattern synthesis by non-uniform spacing and phase control," IEEE Transactions on Antennas and Propagation, vol. 59, no. 12, pp. 4505-4511, 2011.

[9] Ó. Quevedo-Teruel and E. Rajo-Iglesias, "Ant colony optimization in thinned array synthesis with minimum sidelobe level," IEEE Antennas and Wireless Propagation Letters, vol. 5, no. 1, pp. 349-352, 2006.

[10] K. V. Deligkaris, Z. D. Zaharis, D. G. Kampitaki, S. K. Goudos, I. T. Rekanos, and M. N. Spasos, "Thinned planar array design using boolean PSO with velocity mutation," IEEE Transactions on Magnetics, vol. 45, no. 3, pp. 1490-1493, 2009.

[11] R. Karimzadeh Baee, K. Forooraghi, and S. Chamaani, "Conformal array pattern synthesis using a hybrid WARP/2LB-MOPSO algorithm," International Journal of Antennas and Propagation, vol. 2012, Article ID 202906, 7 pages, 2012.

[12] M. A. Panduro and C. Brizuela, "Evolutionary multi-objective design of non-uniform circular phased arrays," International Journal for Computation and Mathematics in Electrical and Electronic Engineering, vol. 27, no. 2, pp. 549-564, 2008.

[13] S.-C. Chu and P.-W. Tsai, "Computational intelligence based on the behavior of cats," International Journal of Innovative Computing, Information and Control, vol. 3, no. 1, pp. 163-173, 2007.

[14] L. Pappula and D. Ghosh, "Linear antenna array synthesis using cat swarm optimization," AEU-International Journal of Electronics and Communications, vol. 68, no. 6, pp. 540-549, 2014.

[15] L. Pappula and D. Ghosh, "Synthesis of thinned planar antenna array using multiobjective normal mutated binary cat swarm optimization," Applied Computational Intelligence and Soft Computing, vol. 2016, Article ID 4102156, 9 pages, 2016.

[16] L. Cen, W. Ser, Z. L. Yu, S. Rahardja, and W. Cen, "Linear sparse array synthesis with minimum number of sensors," IEEE Transactions on Antennas and Propagation, vol. 58, no. 3, pp. 720-726, 2010.

[17] L. Cen, Z. L. Yu, and W. Ser, "Antenna array synthesis in presence of mutual coupling effect for low cost implementation," in Proceedings of the 12th International Symposium on Integrated Circuits (ISIC '09), pp. 360-363, Singapore, December 2009.

[18] X. Yuan, Z. Li, D. Rodrigo et al., "A parasitic layer-based reconfigurable antenna design by multi-objective optimization," IEEE Transactions on Antennas and Propagation, vol. 60, no. 6, pp. 2690-2701, 2012.

[19] D. J. Ludick, R. Maaskant, D. B. Davidson, U. Jakobus, R. Mittra, and D. de Villiers, "Efficient analysis of large aperiodic antenna arrays using the domain Green's function method,"
IEEE Transactions on Antennas and Propagation, vol. 62, no. 4, pp. 1579-1588, 2014.

[20] D. M. Pozar, "Active element pattern," IEEE Transactions on Antennas and Propagation, vol. 42, no. 8, pp. 1176-1178, 1994.

[21] D. F. Kelley and W. L. Stutzman, "Array antenna pattern modeling methods that include mutual coupling effects," IEEE Transactions on Antennas and Propagation, vol. 41, no. 12, pp. 1625-1632, 1993.

[22] Q.-Q. He, B.-Z. Wang, and W. Shao, "Radiation pattern calculation for arbitrary conformal arrays that include mutualcoupling effects," IEEE Antennas and Propagation Magazine, vol. 52, no. 2, pp. 57-63, 2010.

[23] Q.-Q. He, H.-D. He, H. Lan, and Y. Zhang, "An effective calculation method for conformal array mounted on curvedsurface platform," in Proceedings of the 2011 China-Japan Joint Microwave Conference (CJMW '11), pp. 175-178, IEEE, Hangzhou, China, April 2011.

[24] J. H. Liu and J. Lampinen, "A fuzzy adaptive differential evolution algorithm," in Proceedings of the IEEE Region 10 Conference on Computers, Communications, Control and Power Engineering, pp. 606-611, Beijing, China, October 2002. 


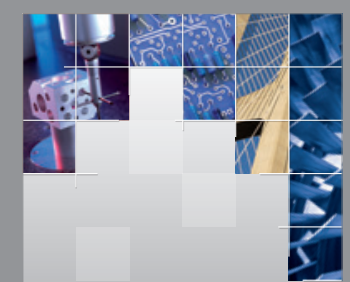

\section{Enfincering}
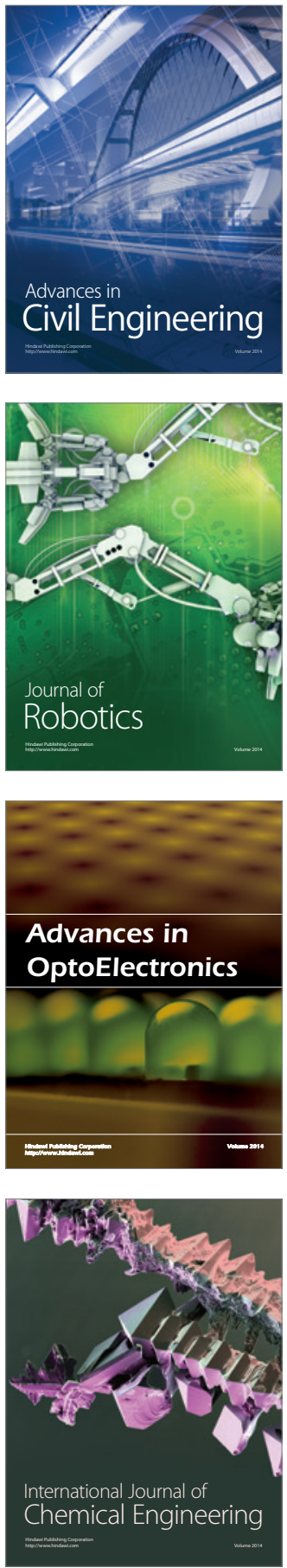

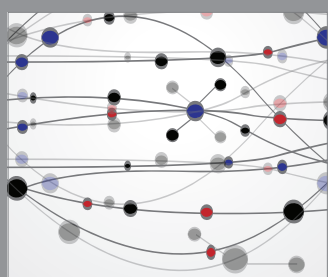

The Scientific World Journal

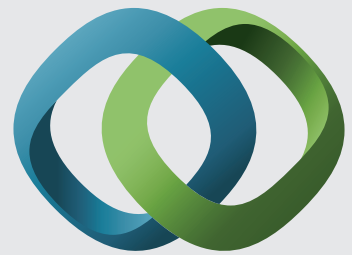

\section{Hindawi}

Submit your manuscripts at

http://www.hindawi.com
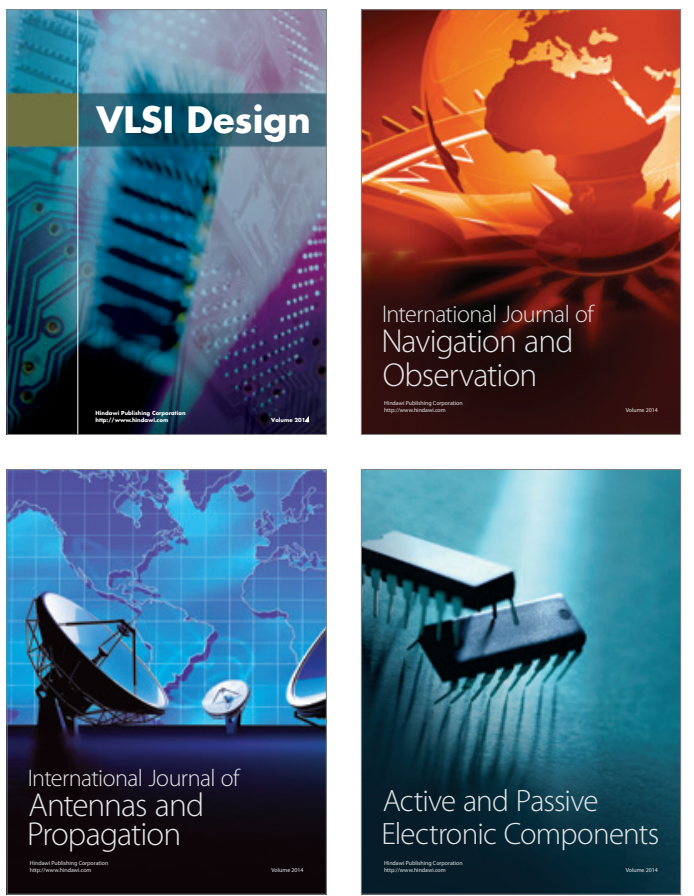
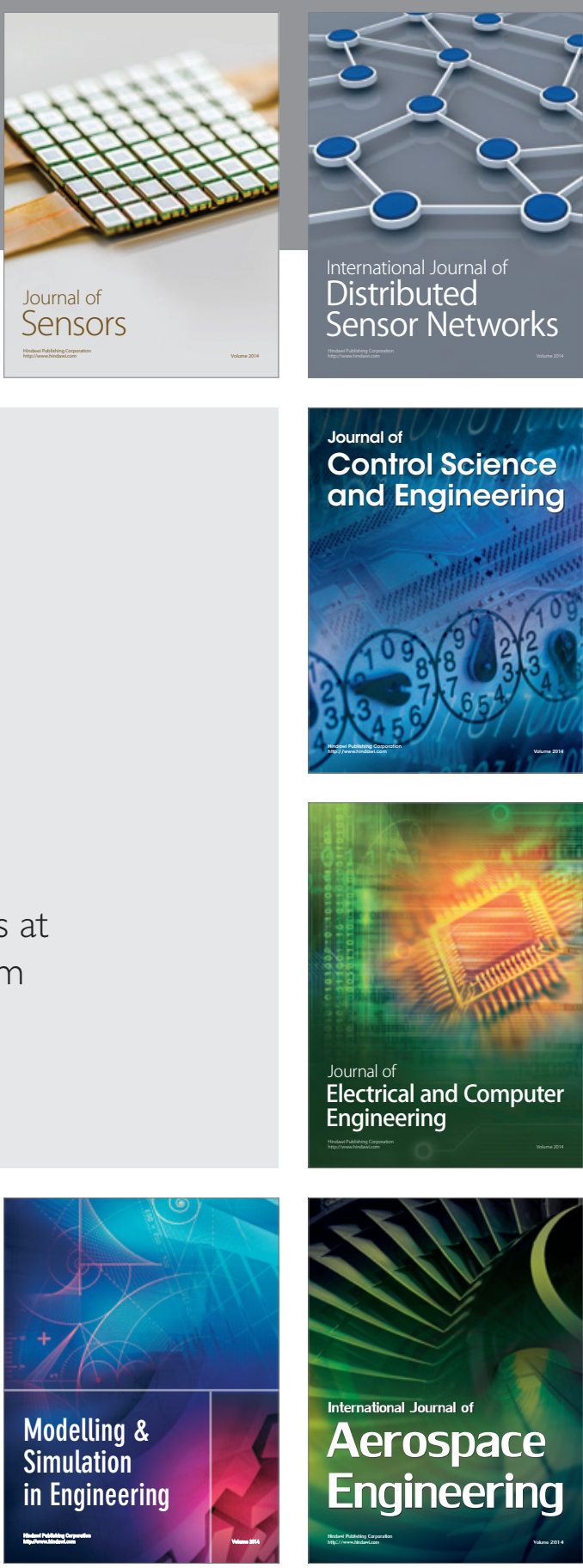

International Journal of

Distributed

Sensor Networks

Journal of

Control Science

and Engineering
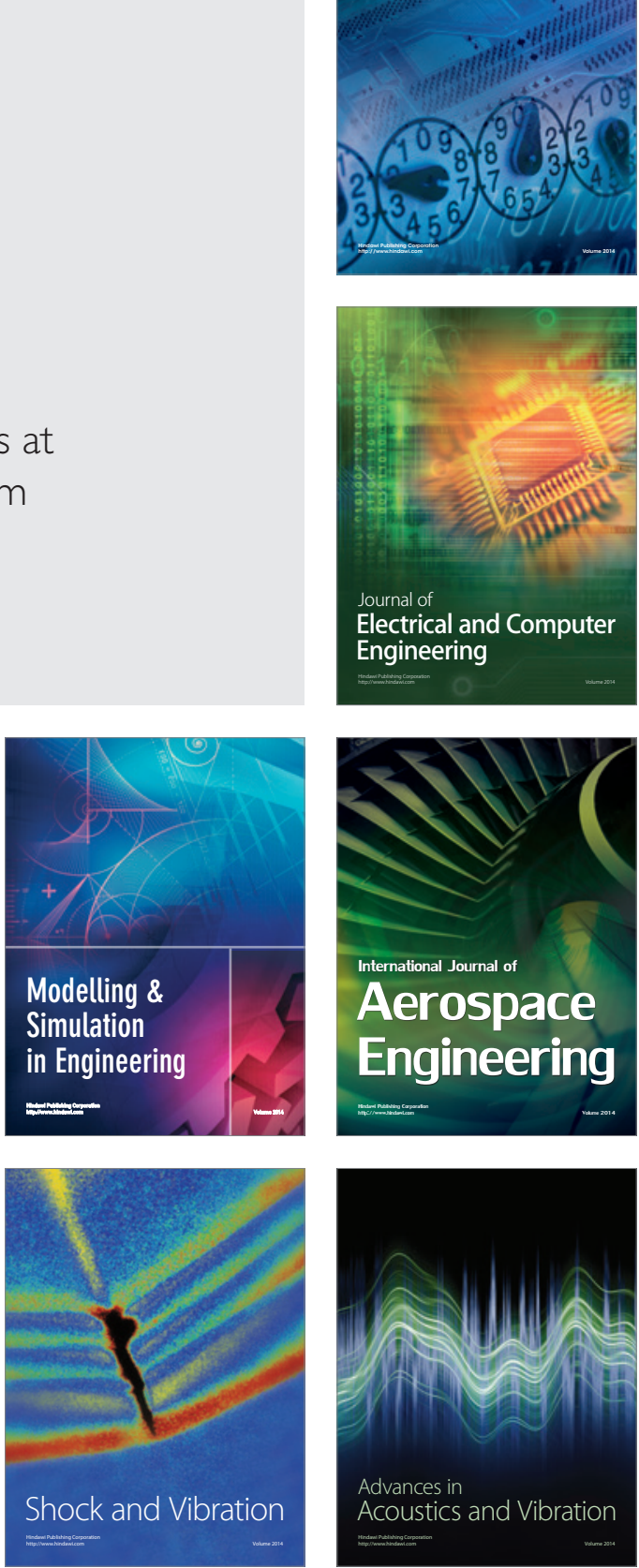